\title{
Narrowing of knee joint space in patients with pseudogout
}

\author{
MICHAEL H. ELLMAN, NANCY L. BROWN, AND BERTRAM LEVIN \\ From the Departments of Medicine and Diagnostic Radiology, Michael Reese Hospital and Medical Center, \\ Chicago, Illinois 60616, USA
}

SUMMARY Abnormalities of joint space in knee roentgenograms were compared among 28 symptomatic patients with pseudogout and 2 control groups consisting of spouses of patients and hospital based patients with knee pain. Joint space narrowing was significantly more frequent in the pseudogout patients than in the 2 control groups. Division of patients and controls into 3 age groups revealed a striking degree of joint space narrowing in the youngest patients with pseudogout. Our study suggests that symptomatic patients with pseudogout have joint destruction beginning at a relatively early age. We speculated that this may reflect concurrence of chondrocalcinosis and another disease such as osteoarthritis.

Pseudogout (calcium pyrophosphate dihydrate disease-CPPD) is a common, chronic arthritic disorder of the elderly. The disease is characterised by the presence of calcium pyrophosphate dihydrate crystals in joint fluid and articular chondrocalcinosis, the roentgenographic demonstration of calcium pyrophosphate dihydrate crystals in cartilage. ${ }^{12}$ The natural history of the sporadic form of CPPD is unknown, though Resnick et $a .^{3}$ described distinctive, severe, and progressive arthritis in CPPD patients compared to osteoarthritic controls. Progressive deterioration of cartilage with development of secondary osteoarthritis and narrowing of the joint space in familial polyarticular CPPD has been graphically demonstrated by Zitnan and Sitaj. ${ }^{4}$ We have attempted to assess destruction of knee cartilage in symptomatic CPPD patients by measuring the joint space in knee roentgenograms and comparing them with a normal control population (spouses of patients) and with an age and sex matched hospital based population with knee pain.

\section{Material and methods}

The age and sex composition of the CPPD patients and the control populations is shown in Table 1. The CPPD patients were referred to a hospital based rheumatology practice because of the chief complaint of knee pain. Twelve patients had acute

Accepted for publication 9 January 1980

Correspondence to Dr M. H. Ellman, Director, Division of Rheumatology, Department of Medicine, Michael Reese Hospital and Medical Center, 2929 Ellis Avenue, Chicago, Illinois 60616, USA.
Table 1 Age and sex composition of CPPD patients, spouses, and knee pain control subjects (see 'Methods')

\begin{tabular}{llll}
\hline & $\begin{array}{l}\text { CPPD } \\
\text { pts }\end{array}$ & $\begin{array}{l}\text { Spouse } \\
\text { controls }\end{array}$ & $\begin{array}{l}\text { Knee pain } \\
\text { controls }\end{array}$ \\
\hline Total number & 28 & 28 & 28 \\
Mean age (years) & $63.4 \pm 10 \cdot 4$ & $61 \cdot 6 \pm 10 \cdot 5$ & $63 \cdot 2 \pm 10 \cdot 1$ \\
Number of males & 18 & 10 & 18 \\
Mean age of males & $60 \cdot 7 \pm 9 \cdot 8$ & $69 \cdot 5 \pm 9 \cdot 5$ & $60 \cdot 3 \pm 9 \cdot 0$ \\
Number of females & 10 & 18 & 10 \\
Mean age of females & $68 \cdot 2 \pm 10.0$ & $57 \cdot 3 \pm 8 \cdot 5$ & $68 \cdot 4 \pm 10.4$ \\
\hline
\end{tabular}

\pm Standard deviation.

or semiacute symptoms at the time of the initial visit. They underwent arthrocentesis and all had calcium pyrophosphate dihydrate crystals in synovial fluid demonstrated by compensated polarised light microscopy. Two of the patients were referred because of knee pain occurring after general surgery. Typical chondrocalcinosis was present on standard anteroposterior, non-weight bearing knee roentgenograms in all 28 patients. A normal control population was obtained by inviting spouses of patients to participate in the study (spouse controls). Another control population, age and sex matched to the CPPD patients, was obtained by culling the 1971 in- and outpatient hospital radiology files for patients with non-weight bearing knee roentgenograms and a history of knee pain (knee pain controls). These were obtained by selecting the first encountered roentgenograms matching the age and sex of the CPPD patients. Roentgenograms were excluded if there was chondrocalcinosis, a recent fracture, or if the films were part of a skeletal survey for malignancy. 
Unanimity among the 3 authors was required for a roentgenogram to be considered positive for chondrocalcinosis. Each author independently measured the vertical joint space of the inner and outer knee compartment at its midline with a millimeter rule, comparing the most narrowed compartment with the adjacent compartment or the identical compartment of the contralateral knee. Some patients had complete joint space collapse and no measurements were required. We were forced to guess the degree of narrowing in an occasional patient when all 4 knee compartments were clearly abnormal. The measurements of the most severely involved compartment were recorded according to the following scale: $1=$ less than half joint space narrowing; $2=$ half narrowing; and $3=$ greater than half narrowing. There was excellent agreement between the 3 authors about the presence and degree of joint space narrowing.

Standard biochemical and parathyroid and thyroglobulin stimulating hormone assays were performed on the CPPD patients and their spouses (reported in detail elsewhere). ${ }^{5}$

\section{Results}

Nineteen of the 28 CPPD patients had narrowing of the knee joint space compared with 4 out of 28 spouses $(P<0.005)$ (chi-square test) and 10 out of 28 controls with knee pain $(P<0 \cdot 05)$.

The CPPD patients and the 2 control populations were divided into 3 groups based on age, and the knee roentgenograms were compared (Fig. 1). The CPPD patients had more frequent joint space narrowing in all age groups than did the spouses. There was more frequent narrowing of the joint space only in the youngest CPPD patient group than in the controls with knee pain. Similar results were obtained when the extent of joint space narrowing was compared among the 3 groups in the 3 age categories. The mean degree of joint space narrowing increased with age.

There was little correlation (correlation coefficient, $r=0 \cdot 16)$ between the extent of chondrocalcinosis (minimal=linear stippled calcification limited to fibro or hyaline cartilage; moderate = calcification of fibro and hyaline cartilage; marked = same as $2+$ synovial or extra-articular, linear stippled calcification) and degree of joint space narrowing.

All abnormal roentgenographic findings except for the chondrocalcinosis were compatible with the changes of osteoarthritis.

We found primary hyperparathyroidism in 2 of our patients; another patient had hyperparathyroidism secondary to mild, chronic renal failure.

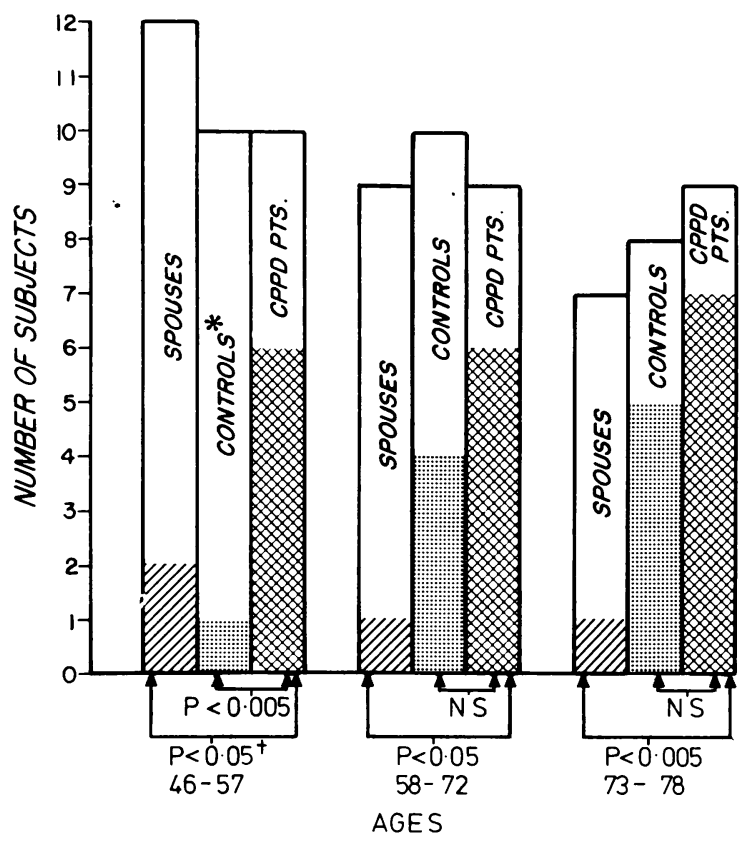

Fig. 1 Number of subjects, divided into age groups, with joint space narrowing (shaded areas): ${ }^{*} k n e e$ pain controls (see Methods); †chi-square test; NS=not significant.

An additional patient had raised parathyroid hormone levels with normal serum calcium and urea nitrogen values but persistent hypomagnesaemia. None of the spouse controls had similar abnormalities.

\section{Discussion}

CPPD is a relatively newly described rheumatic entity and the natural course of the disease is unkrown. The various clinical expressions of CPPD and its poorly defined relationship to oeteoarthritis make it a difficult disease to study. ${ }^{26-8}$ A comprehensive review by Resnick et al. ${ }^{3}$ showed that of 154 knees with chondrocalcinosis in 80 patients 97 had arthropathy (56 patients). Roentgenographic abnormalities of knees were 3.6 times more common in CPPD patients than in a control group consisting of patients undergoing a skeletal survey for either possible metastatic disease or for minor articular complaints. $^{3}$ Martel et al. $^{9}$ found structural joint abnormalities resembling severe osteoarthritis in the majority of their CPPD patients. Progressive joint destruction in familial CPPD has been demonstrated in patients in Czechoslovakia ${ }^{4}$ and Chile. ${ }^{10}$ 
We have shown that CPPD patients selected for knee pain have more frequent joint space narrowing than a normal control population and a control group having knee roentgenograms for knee pain. The CPPD patients have a strikingly earlier age of onset of knee joint abnormalities than either control group. Six of 10 CPPD patients under the age of 57 already had joint space narrowing on knee roentgenograms compared with 2 out of 12 spouse controls and 1 out of 10 controls with knee pain (Fig. 1).

The explanation for the earlier onset and progressive joint space narrowing in the symptomatic CPPD patients is unknown. It is possible that our study based on patients referred to hospital has selected those with the most severe CPPD. However, chondrocalcinosis as such may be unassociated with arthritis. In a previous study we found no significant differences in arthritic complaints between elderly subjects with and without chondrocalcinosis. ${ }^{11}$ Indeed, asymptomatic chondrocalcinosis may be the most common subset of CPPD. ${ }^{8}$

We postulate that the clinical expression of CPPD may require the presence of chondrocalcinosis and another factor such as osteoarthritis, joint trauma, postoperative electrolyte changes, ${ }^{12}$ or a metabolic disease, especially hyperparathyroidism. ${ }^{13}$ Severe joint destruction has been reported in patients with concurrent osteoarthritis and CPPD. ${ }^{14}{ }^{15}$ We found significant metabolic abnormalities in 4 of our CPPD patients but in none of the spouse controls. Although not studied systematically, several of our younger patients with CPPD were referred to us with knee pain occurring after trauma.

It is conceivable that diseases such as osteoarthritis that are associated with premature cartilage fibrillation and fragmentation lead when combined with chondrocalcinosis to the release of calcium pyrophosphate dihydrate crystals into the joint cavity. The crystals invoke an inflammatory and often persistent synovitis that promotes accelerated cartilage destruction. These patients come to the attention of the clinician and comprise a sutset of CPPD with severe disease.
This work was supported by a grant from the Illinois Chapter of the Arthritis Foundation.

\section{References}

1 McCarty D J, Haskin M E. The roentgenographic aspects of pseudogout (articular chondrocalcinosis). An analysis of 20 cases. AJR 1963; 90: 1248-57.

2 McCarty D J, Pseudogout, articular chondrocalcinosis: Calcium pyrophosphate deposition diseases. In: Hollander J L, McCarty D J, eds. Arthritis and Allied Conditions, 8 ed. Philadelphia: Lea and Febiger, 1972; 1140-60.

3 Resnick D, Niwayama G, Goergen T G, et al. Clinical radiographic and pathologic abnormalities in calcium pyrophosphate dihydrate deposition disease (CPPD): Pseudogout. Radiology 1977; 122: 1-15.

4 Zitnan D, Sitaj S. Natural course of articular chondrocalcinosis. Arthritis Rheum 1976; 19: 369-90.

5 Ellman M H, Brown N L, Porat A. Laboratory investigations in pseudogout patients. $J$ Rheumatol 1980; 7: $77-81$.

6 Silcox D C, McCarty D J. Elevated inorganic pyrcphosphate concentrations in synovial fluids in osteoarthritis and pseudogout. $J$ Lab Clin Med 1974; 83: 518-31.

7 Howell D S, Muniz O, Pita J C, Enis J E. Pyrophosphate release by osteoarthritis cartilage incubates. Arthritis Rheum 1976; 19: 488-94.

8 McCarty D J. Calcium pyrophosphate dihydrate crysta deposition disease-1975. Arthritis Rheum 1976; 19: 275 85.

9 Martel W, Chamption C K, Thompson G R, Carter T L. A roentgenologically distinctive arthropathy in some patients with the pseudogout syndrome. $A J R, 1970$; 109: 587-605.

10 Reginato A J. Articular chondrocalcinosis in the Chiloé Islanders. Arthritis Rheum 1976; 19: 395-404.

11 Ellman M H, Levin B. Chondrocalcinosis in elderly persons. Arthritis Rheum 1975; 18: 43-7.

12 O'Duffy J D. Clinical studies of acute pseudogout attacks. Comments on prevalence, predispositions, and treatment. Arthritis Rheum 1976; 19: 349-52.

13 Hamilton E B D. Diseases associated with CPPD deposition disease. Arthritis Rheum 1976; 19: 353-7.

14 Gerster J C, Vischer T L, Boussina T, Fallet G H. Joint destruction and chondrocalcinosis in patients with generalised osteoarthrosis. Br Med J 1975; iv, 684.

15 Gerster J C, Vischer T L, Fallet G H. Destructive arthrcpathy in generalised osteoarthritis with articular chondrocalcinosis. $J$ Rheumatol 1975; 1 : 265-9. 